\title{
Article \\ Intensive Rehabilitation Program in Arterial Occlusive Disease Patients
}

\author{
Luca Marin ${ }^{1,2,3,4}{ }^{(0)}$, Nicola Lovecchio ${ }^{1}{ }^{(0}$, Adam Kawczynski ${ }^{5}\left(\mathbb{D}\right.$, Massimiliano Febbi $^{2,4}$, Dario Silvestri ${ }^{6}$, \\ Vittoria Carnevale Pellino ${ }^{1,7}\left(\mathbb{D}\right.$, Raffaele Gibellini ${ }^{3}$ and Matteo Vandoni ${ }^{1, *} \mathbb{B}$
}

1 Laboratory of Adapted Motor Activity (LAMA), Department of Public Health, Experimental Medicine \& Forensic Science, University of Pavia, 27100 Pavia, Italy; luca.marin@unipv.it (L.M.); nicola.lovecchio@unipv.it (N.L.); vittoria.carnevalepellino@unipv.it (V.C.P.)

2 Department of Rehabilitation, Faculty of Medicine, University of Ostrava, 70103 Ostrava, Czech Republic; massimilianofebbi@gmail.com

3 Department of Rehabilitation, "Città di Pavia" Hospital, 27100 Pavia, Italy; ragibell@gmail.com

4 Laboratory for Rehabilitation, Medicine and Sport (LARMS), 00133 Rome, Italy

5 Department of Paralympics Sports, University School of Physical Education, 51-617 Wrocław, Poland; kawczynski.a@gmail.com

6 Department of Research, Asomi College of Sciences, 2080 Marsa, Malta; direttore@asomi-osteopatia.com

7 Department of Industrial Engineering, University of Tor Vergata, 00133 Rome, Italy

* Correspondence: matteo.vandoni@unipv.it; Tel.: +39-0382-987166

Citation: Marin, L.; Lovecchio, N.; Kawczynski, A.; Febbi, M.; Silvestri, D.; Pellino, V.C.; Gibellini, R.; Vandoni, M. Intensive Rehabilitation Program in Arterial Occlusive Disease Patients. Appl. Sci. 2021, 11, 1184. https://doi.org/10.3390/ app11031184

Academic Editor: Mark King

Received: 14 December 2020

Accepted: 23 January 2021

Published: 28 January 2021

Publisher's Note: MDPI stays neutral with regard to jurisdictional claims in published maps and institutional affiliations.

Copyright: (c) 2021 by the authors. Licensee MDPI, Basel, Switzerland. This article is an open access article distributed under the terms and conditions of the Creative Commons Attribution (CC BY) license (https:// creativecommons.org/licenses/by/ $4.0 /)$.

\begin{abstract}
Peripheral arterial occlusive disease (PAOD) limits walking efficiency and distance. The main disabling symptom is vasculopathy that leads to claudicatio intermittens and limits walking efficiency. Stretching techniques are effective in treatments for retractions and are used to improve flexibility of triceps surae and range of motion of the ankle. The aim of this study is to evaluate the effects of a combined walking and stretching program on walking efficiency in elderly PAOD patients. Seventy patients with PAOD stage II of the Leriche-Fontaine classification were randomly assigned into a stretching group (SG) or conditioning group (CG). Both groups participated in an eleven-day intensive rehabilitation program based on walking and strength training, while the SG performed five extra sessions of stretching. Walking autonomy was assessed through treadmill and overground tests with the recording of initial and absolute pain. A univariate ANOVA analysis was applied for the differences between the initial and final outcomes. Walking autonomy improved in both groups $(p<0.01)$, while only the SG improved flexibility (from $-14.0 \pm 8.1$ to $-10.3 \pm 8.3 \mathrm{~cm} ; p<0.01$ ). An intensive eleven-day rehabilitation program based on walking, strength, and stretching exercises is effective to improve the onset and the delay of pain during walking in patients with PAOD.
\end{abstract}

Keywords: PAOD; exercise; flexibility; walking autonomy

\section{Introduction}

Peripheral arterial occlusive disease (PAOD) is a syndrome that showsdifferent nosological symptoms due to the reduction of blood flow in the lower limbs district [1,2]. The main disabling symptom is vasculopathy, since the poor oxygenation of calf muscles leads to altered gait patterns called claudicatio intermittens [3]. This painful condition limits walking efficiency (speed and distance covered without rest), which is a crucial aspect of daily living activity: general quality of life is ipso-facto negatively affected. PAOD prevalence is steadily increasing worldwide (between $3 \%$ and $12 \%$ ) and is assuming social relevance, since there are 27 million European people suffering from vasculopathy [4]. PAOD development exhibits a progressive incidence with age [5] that could lead to increased cardiac and cerebrovascular mortality [6]. Clinical approaches vary from surgical to pharmacological treatments, although rehabilitation training programs also meet the recommendations from the American College of Cardiology and the American Heart Association [7]. In particular, supervised walking training programs are more effective 
than plans based on walking or simple exercise recommendations [4,8]. Patterson et al. [9] showed that a hospital-based program was more effective than a home-based program because supervised plans were able to better focus on the patient needs and to reduce drop-out rates [10]. Other studies have focused on lower limbs rehabilitation protocol with walking or mixed training programs [8,11] (both aerobic and resistance), but information about muscular pain/retraction is still missing. In fact, clinical practice has suggested that a lot of patients suffer pain in the lumbar region associated with a retraction of the muscles [12]. This framework is probably a driver toward claudication [13]: calf muscles stiffness reduces, for example, the ankle dorsal flexion and then increases the risk of fall during the clearance of the feet [14]. Stretching techniques are effective in treatments for retractions and are commonly used to improve flexibility of triceps surae and range of motion of the ankle [15]. Within this complex pathology, static analytical stretching is recognized as a golden practice [3], with an optimal duration per session lasting from 5 to $30 \mathrm{~min}[14,15]$. In addition, Crowther et al. (2008) [11] investigated the effects of a 12-month supervised exercise program (based on a progression of walking phase on a treadmill) on the general functionality of lower limbs in PAOD old subjects: the walking time performance was augmented $120 \%$. Probably, the compromised flexibility of elderly subjects [16] benefits from stretching exercises (improving hip and ankle range of motion), which, in turn, improves walking speed and gait length $[17,18]$. From a functional point of view, improved flexibility in lower limbs and all its components (muscles, tendons, and joints) could generally improve the gait [15]. In this view, a specific stretching training of lower limb muscles could positively affect the process of rehabilitation in PAOD patients. According to this rationale, the aim of the present study is to evaluate the effects of a specific stretching program on walking performance in elderly PAOD patients.

\section{Materials and Methods}

\subsection{Subject}

Seventy patients (14 females) (mean age $71.5 \pm 8.2$ years, weight $73.7 \pm 9.4 \mathrm{~kg}$, height $1.71 \pm 4.3$, and Body Mass Index $25.3 \pm 2.1$ ) with PAOD stage II of the Leriche-Fontaine classification [19] were freely recruited in hospital setting (Città di Pavia, Pavia, Italy) and were randomly divided into two groups: the stretching group (SG, $n=37$ ) and the conditioning group (CG, $n=33$ ). Both groups followed the same rehabilitation program (as specified below), while the SG performed five extra sessions of stretching exercises. Inclusion criteria were age $\geq 65$ years, stability of the lesion, and no arterial bypass, heart disease, or neurological affection that could hinder adherence to the protocol. Exclusion criteria were as follows: uncertain of patient's cardiac status (check-up older than 6 months or not done), severe or unstable cardiopulmonary pathologies, contra-indications to the ability to participate in strength training, especially aortic dissection and atheromatous emboli or stenosis at the prethrombotic state without potential collateral consequences and critical lower limb ischemia. In particular, the vascular therapy (antiplatelet) was kept stable and no major modulations were introduced into the medical treatment.

The study protocol (supervised by a clinician), including each feature of the experimental design, was approved by the ethical boards of the hospital in accordance with the "World Medical Association Declaration of Helsinki" as revised in 1983. All participants provided a written informed consent before participation in the study and were free to withdraw their participation at any time.

\subsection{Procedure}

The flow of the pre/post protocol is showed in Figure 1. 


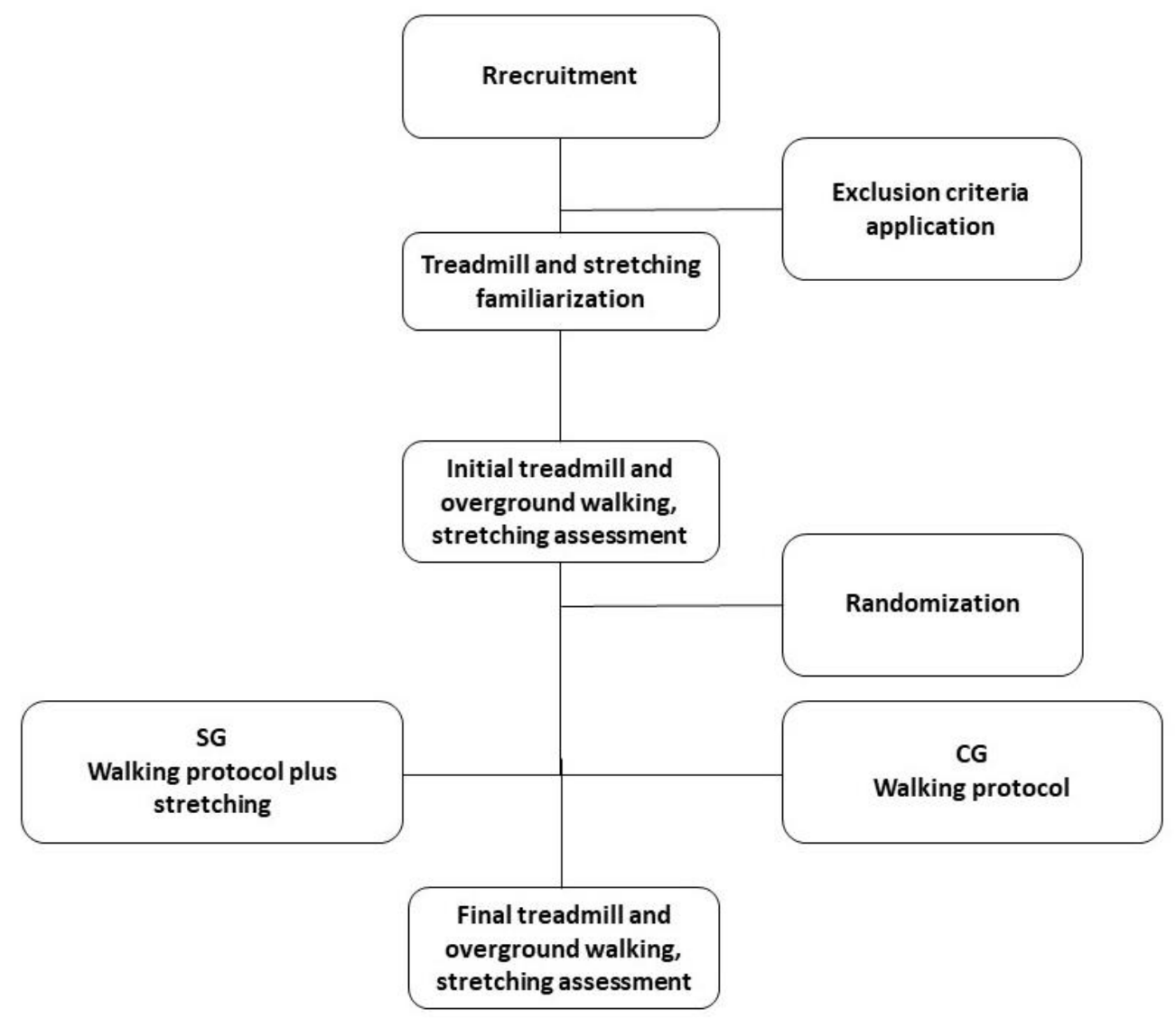

Figure 1. Study procedures flow chart. SG: stretching group; CG: conditioning group.

In brief, all of the subjects performed two assessments: at baseline (pre), the day after the admission to the hospital; and at the end of the hospitalization (post), one day before the hospital discharge. All procedures were administrated by the same operator in a blinded modality.

Walking autonomy was assessed through a test on a treadmill (defined as 'treadmill test' in Figure 1) and by adopting an overground walking trial (defined as 'walking test') [19,20]. During treadmill and overground trials, the initial claudication pain (ICP; [19]) and the absolute claudication pain (ACP; [19]) were detected by measuring the time (s) elapsed from the beginning of the test: in practice, the period of time for the initial pain detection to occur and the time at which the test was stopped due to insufferable pain. More precisely, four indexes were obtained: treadmill initial and absolute claudication pain (TICP, TACP, respectively) and walking initial and absolute claudication pain (WICP, WACP, respectively).

\subsection{Treadmill Test}

Before the treadmill test, participants were familiarized with walking on the same treadmill (Ergo Run Medical 8, Daum Electronic GmbH, Fürth, Germany) used for the training.

The evaluation was carried out at a speed of $3.2 \mathrm{~km} / \mathrm{h}$ [7], with an initial null inclination and then increasing by $2 \%$ every $2 \mathrm{~min}$ for a maximum duration of $12 \mathrm{~min}$ or to the achievement of absolute pain, as described elsewhere [13]. The trials might be interrupted at the appearance of one or more criteria described below as 'termination test criteria'. Following these criteria, participants were considered asymptomatic if ICP did not occur until the 12th minute of the test. The same operator measured the performance by time (s) before the onset of the TICP and TACP.

\subsection{Walking Test}

The walking test was performed over ground at a self-chosen pace on a $20 \mathrm{~m}$ plane path. The test usually [19] lasted until the onset of ACP, with a maximum duration of 
$12 \mathrm{~min}$, which was considered the asymptomatic stopping point. It could be interrupted at the appearance of one or more criteria described as 'termination test criteria'. The same operator measured the walking distance in time (in seconds) before the onset of the WICP and WACP.

\subsection{Termination Test Criteria}

As described in literature [20], criteria to stop the test were heart rate $>85 \%$ of maximum predicted, cardiovascular symptoms (pallor, forehead sweating, cyanosis, dyspnea, or tachypnea), osteo-articular intense pain, and/or voluntary interruption according to the patient's request.

\subsection{Flexibility Test}

The sit-and-reach test was used because it provides a global measure of hamstring, hip, and lower back flexibility [21]. The participants sit with their bare feet pressed against a wooden box. Knees were extended, and the right hand was positioned over the left. Participants were then asked to slide the hand over a ruler located over the box as far as possible avoiding bounce action. Each subject performed two trials, and the best trial was used for analysis.

\section{Rehabilitation Protocol}

The stretching and conditioning groups followed an intensive eleven-day rehabilitation protocol (eleven days is the typical period of our clinic for the hospitalization of PAOD patients) based on two daily sessions (from 8:00 to 10:00 and from 15:00 to 17:00) of walking on the treadmill, while a series of proprioceptive exercises and strength conditioning were performed only during the morning appointment. The treadmill walking sessions consisted of four bouts that lasted 70\% of the time of TICP (recorded during the initial test), observing three minutes of rest between repetition. Strength exercises consisted of 20 min of lower extremity strength training: three sets of ten repetitions (rest $180 \mathrm{~s}$ between the sets) composed of equipment-free leg extensions, chair-raises, calf-raises, and toe-raise exercises (a plantar flexion exercise where participants assumed a tip-toe position and lowered themselves ten times consecutively) followed by $10 \mathrm{~min}$ of active exercises for ankle and metatarsal phalangeal joints.

\subsection{Stretching Protocol}

In accordance with the literature, stretching analytical technique was used [15] to perform the adjunctive five sessions of stretching of the calf muscles (Figures 2 and 3). The duration of the stretching session (showed in Table 1) was defined on the basis of clinical evidence [14,22], as described in Table 1 . The activity was performed under the supervision of a physiotherapist in order to guarantee the maintenance of correct body arrangement. Two exercises were alternated during the period.

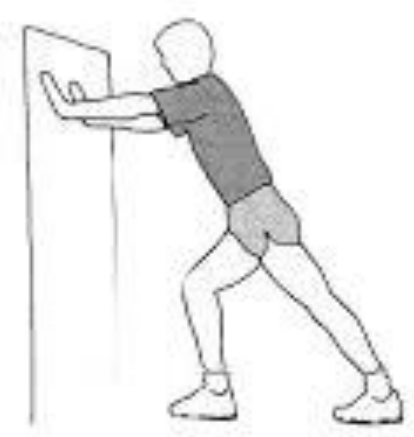

Figure 2. Calf muscle stretching exercise 1. 


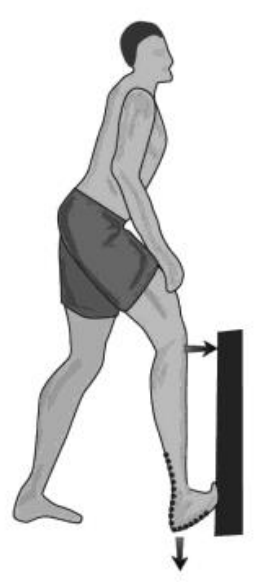

Figure 3. Calf muscle stretching exercise 2.

Table 1. Stretching protocol.

\begin{tabular}{cccc}
\hline $\begin{array}{c}\text { Series } \\
\boldsymbol{N}\end{array}$ & $\begin{array}{c}\text { Repetition } \\
\boldsymbol{n}\end{array}$ & $\begin{array}{c}\text { Time } \\
\text { (s) }\end{array}$ & $\begin{array}{c}\text { Recovery } \\
\text { (s) }\end{array}$ \\
\hline 1 & 3 & 30 & 30 \\
2 & 3 & 45 & 45 \\
3 & 4 & 45 & 45 \\
4 & 4 & 60 & 60 \\
5 & 5 & 60 & 60 \\
\hline
\end{tabular}

\subsection{Statistical Analysis}

The ICP and ACP data were analyzed at the initial time (T0) and at the end of the study (T1).

Results within the groups were analyzed using a t-test analysis for paired samples. The effects on each parameter were subsequently quantified considering the difference between the values recorded at $\mathrm{T} 1$ and $\mathrm{T} 0(\Delta)$. A univariate ANOVA analysis was applied to the $\Delta$ for the calculation of the differences between the groups (SG and CG) over time. The calculation of the post hoc values was obtained through the Tukey method. Data were analyzed with the IBM SPSS Statistics for Windows, Version 21.0. Armonk, NY, USA, IBM Corp. The values were expressed as means \pm standard deviation, and the significance level was set at $p<0.05$.

\section{Results}

A total of 70 patients (14 females) completed the study.

Hospitalization time was similar between the groups $(11.2+1.3$ days for SG vs. $11.4+1.4$ days for CG). Walking autonomy was improved in both groups (Table 2). In particular, the test on the ground revealed $63 \%$ and $75 \%$ increases in time for ICP (SG and CG, respectively), while the time for ACP increased by $73 \%$ and $71 \%$ in SG and CG, respectively. The treadmill trials showed that the amelioration was $63 \%$ in the SG and $58 \%$ in the CG for ICP, while there was an increase of $71 \%$ and $63 \%$ of ACP in SG and CG, respectively. Finally, SG improved flexibility from $-14.0 \pm 8.1$ to $-10.3 \pm 8.3 \mathrm{~cm}(p<0.01)$, while the CG changed from $-16.2 \pm 4.9$ to $-15.0 \pm 6.8$ (ns). Post hoc values obtained through the Tukey method revealed no differences in any outcome between the groups. 
Table 2. Pain outcomes during walking tests.

\begin{tabular}{|c|c|c|c|c|}
\hline \multirow{2}{*}{$\begin{array}{c}\text { Outcome } \\
\text { WICP }\end{array}$} & \multicolumn{2}{|c|}{ SG } & \multicolumn{2}{|c|}{ CG } \\
\hline & $190.4 \pm 97.5$ & $302.7 \pm 113.5$ & $165.6 \pm 77.1$ & $222.8 \pm 77.1^{*}$ \\
\hline WACP & $292.6 \pm 118.3$ & $400.7 \pm 135.3 *$ & $251.8 \pm 113.1$ & $355.7 \pm 146.4$ \\
\hline TICP & $188.0 \pm 80.4$ & $300.5 \pm 100.5$ * & $181.8 \pm 63.3$ & $314.2 \pm 109.1 *$ \\
\hline TACP & $283.9 \pm 105.7$ & $402.5 \pm 113.9 *$ & $256.4 \pm 82.2$ & $408.9 \pm 116.0 *$ \\
\hline
\end{tabular}

* Significant difference $(p<0.05)$. WICP: walking initial claudication pain; WACP: walking absolute claudication pain; TICP: treadmill initial claudication pain; TACP: treadmill absolute claudication pain; SG: stretching group; and CG: conditioning group. All values are expressed in seconds.

\section{Discussion}

PAOD is a condition that limits walking efficiency and distance. Retraction of the muscles combined with a dynamic alteration of the step could be due to claudication. Stretching techniques are effective in treatments for retractions of muscles and are used to improve flexibility of the calf and mobility of the ankle, but their use is not usually contemplated in vascular protocol rehabilitation. The aim of this study was to evaluate the effects of stretching on walking performance in PAOD patients, especially on the onset of pain during walking. After eleven days of an intensive rehabilitation program based on walking, strength training, and stretching (the latter only in SG), our results showed an improvement in ICP and ACP in walking overground and treadmill performance in both groups.

In accordance with Gardner et al. and Hamburg et al. [13,23], our results confirmed the use of treadmill exercise in the rehabilitation program to ameliorate ICP and ACP. Other studies have highlighted that changes in ICP and ACP due to walking rehabilitation program were related to changes in the enzymatic activity $[7,8]$ and to the size/numbers of mitochondria within the myofibers [9]: these positive changes allowed for more efficient utilization of oxygen. We observed a lack of previous data finding an effect of overground walking (in absence of pain), probably due to the difficulty in controlling walking speed. In our study, we showed a similar extent in ICP and ACP values between the two different modalities of walking. Thus, we can affirm that walking overground, when it is possible, could be introduced into rehabilitation programs both in hospital and in subsequent home-based settings. To better assess rehabilitation protocols, further analysis is needed to investigate the spatiotemporal parameters of gait during walking overground or on treadmill (and also with different equipment).

Bisconti et al. [24] showed that stretching exercises produced improvements in blood pressure, arterial stiffness and vascular function, suggesting local and systemic cardiovascular adjustments. So, the introduction of the stretching routine in our PAOD rehabilitation program could have led patients to enhancing their cardiovascular function and to delaying pain onset during walking $[17,18]$.

In accordance to Hortobágyi et al. [25], strength training produced gains in static and dynamic balance and in loading response during the gait cycle. Our program included free-weight lower extremity strength exercises that are not typical of PAOD rehabilitation but that could have positively influenced the results in walking autonomy.

We are conscious that our study had some limitations. Firstly, the small sample of both groups is mainly due to the one to one method of treating subjects. We also did not measure the dynamics of the step, which could help to better describe the effects of the stretching on walking technique: gait analysis after a stretching protocol could clarify the physical outcomes. Moreover, the use of the ankle-brachial pressure index at rest (ABI) or after the exercise session could have helped in understanding the real extent of benefits derived from the adjunctive stretching protocol. Finally, we are conscious that a treadmill training program is difficult to perform in a home-based context for elderly patients, but it could be replaced as bouts of overground walking. Besides these aspects, the strength of our study is the easy and quickly executed protocol that meets the full compliance of patients in a hospital context. Moreover, our results on ICP and ACP time confirmed the 
importance of a multitask approach with PAOD patients [26]. In fact, in a limited time of treatment (eleven days), patients significantly improved walking autonomy on treadmill and overground. The extent of our results translated to the everyday life of patients can modify general autonomy and quality of life.

\section{Conclusions}

An intensive eleven-day rehabilitation program based on walking [13,23], strength, and stretching exercises is effective at improving the onset and the delay of pain during walking in patients with PAOD. Further investigations are needed to establish which dose and typology of stretching are more effective in order to better contrast PAOD symptoms.

Author Contributions: L.M.: Investigation, Conceptualization, Visualization, and Methodology; N.L.: Methodology, Formal Analysis, Data Curation, and Writing-Original Draft Preparation; A.K.: Review and Editing and Formal Analysis; M.F.: Visualization and Investigation; D.S.: Visualization and Investigation; V.C.P.: Investigation and Writing-Original Draft Preparation; R.G.: Conceptualization, Supervision, and Project Administration; M.V.: Methodology, Writing-Review and Editing, Supervision, and Project Administration. All authors have read and agreed to the published version of the manuscript.

Funding: This research received no external funding.

Institutional Review Board Statement: Ethical review and approval were waived for this study, due to normal clinical procedure application during the hospitalization.

Informed Consent Statement: Informed consent was obtained from all subjects involved in the study.

Data Availability Statement: The data presented in this study are available on request from the corresponding author. The data are not publicly available due to privacy reasons.

Conflicts of Interest: The authors declare no conflict of interest.

\section{References}

1. Warburton, D.E.R.; Nicol, C.W.; Bredin, S.S.D. Health benefits of physical activity: The evidence. CMAJ 2006, 174, 801-809. [CrossRef]

2. Pedersen, B.K.; Saltin, B. Exercise as medicine-Evidence for prescribing exercise as therapy in 26 different chronic diseases. Scand. J. Med. Sci. Sports 2015, 25, 1-72. [CrossRef]

3. Muller, M.D.; Reed, A.B.; Leuenberger, U.A.; Sinoway, L.I. Physiology in Medicine: Peripheral arterial disease. J. Appl. Physiol. 2013, 115, 1219-1226. [CrossRef] [PubMed]

4. Norgren, L.; Hiatt, W.R.; Dormandy, J.A.; Nehler, M.R.; Harris, K.A.; Fowkes, F.G.R.; Rutherford, R.B. Inter-Society Consensus for the management of peripheral arterial disease (TASC II). Int. Angiol. 2007, 26, 82-157. [CrossRef]

5. Leng, G.C.; Lee, A.J.; Fowkes, F.G.R.; Whiteman, M.; Dunbar, J.; Housley, E.; Vaughan Ruckley, C. Incidence, natural history and cardiovascular events in symptomatic and asymptomatic peripheral arterial disease in the general population. Int. J. Epidemiol. 1996, 25, 1172-1180. [CrossRef] [PubMed]

6. Artigao-Rodenas, L.M.; Carbayo-Herencia, J.A.; Divisón-Garrote, J.A.; Gil-Guillén, V.F.; Massó-Orozco, J.; Simarro-Rueda, M.; Molina-Escribano, F.; Sanchis, C.; Carrión-Valero, L.; López de Coca, E.; et al. Framingham risk score for prediction of cardiovascular diseases: A population-based study from southern Europe. PLoS ONE 2013, 8, e73529. [CrossRef]

7. Hirsch, A.T.; Haskal, Z.J.; Hertzer, N.R.; Bakal, C.W.; Creager, M.A.; Halperin, J.L.; Hiratzka, L.F.; Murphy, W.R.C.; Olin, J.W.; Puschett, J.B.; et al. ACC/AHA 2005 guidelines for the management of patients with peripheral arterial disease (lower extremity, renal, mesenteric, and abdominal aortic): Executive summary a collaborative report from the American Association for Vascular Surgery/Society for Vas. J. Am. Coll. Cardiol. 2006, 47, 1239-1312. [CrossRef]

8. Cousin, A.; Popielarz, S.; Wieczorek, V.; Tiffreau, V.; Mounier-Vehier, C.; Thevenon, A. Impact of a rehabilitation program on muscular strength and endurance in peripheral arterial occlusive disease patients. Ann. Phys. Rehabil. Med. 2011, 54, 429-442. [CrossRef]

9. Patterson, R.B.; Pinto, B.; Marcus, B.; Colucci, A.; Braun, T.; Roberts, M. Value of a supervised exercise program for the therapy of arterial claudication. J. Vasc. Surg. 1997, 25, 312-318, discussion 318-9. [CrossRef]

10. Bendermacher, B.L.W.; Willigendael, E.M.; Teijink, J.A.W.; Prins, M.H. Supervised exercise therapy versus non-supervised exercise therapy for intermittent claudication. Cochrane Database Syst. Rev. 2006, CD005263. [CrossRef]

11. Crowther, R.; Spinks, W.; Leicht, A.; Sangla, K.; Quigley, F.; Golledge, J. Effects of a long-term exercise program on lower limb mobility, physiological responses, walking performance, and physical activity levels in patients with peripheral arterial disease. JVS 2008, 47, 303-309. [CrossRef] [PubMed] 
12. Gahier, M.; Hersant, J.; Hamel, J.F.; Sempore, Y.; Bruneau, A.; Henni, S.; Abraham, P. A Simple Scale for Screening Lower-Extremity Arterial Disease as a Possible Cause of Low Back Pain: A Cross-sectional Study Among 542 Subjects. J. Gen. Intern. Med. 2020, 35, 1963-1970. [CrossRef] [PubMed]

13. Gardner, A.W.; Katzel, L.I.; Sorkin, J.D.; Bradham, D.D.; Hochberg, M.C.; Flinn, W.R.; Goldberg, A.P. Exercise rehabilitation improves functional outcomes and peripheral circulation in patients with intermittent claudication: A randomized controlled trial. J. Am. Geriatr. Soc. 2001, 49, 755-762. [CrossRef]

14. Radford, J.A.; Burns, J.; Buchbinder, R.; Landorf, K.B.; Cook, C. Does stretching increase ankle dorsiflexion range of motion? A systematic review. Br. J. Sports Med. 2006, 40, 870-875, discussion 875. [CrossRef] [PubMed]

15. Young, R.; Nix, S.; Wholohan, A.; Bradhurst, R.; Reed, L. Interventions for increasing ankle joint dorsiflexion: A systematic review and meta-analysis. J. Foot Ankle Res. 2013, 6, 46. [CrossRef]

16. Shephard, R.J.; Berridge, M.; Montelpare, W. On the generality of the "sit and reach" test: An analysis of flexibility data for an aging population. Res. Q. Exerc. Sport 1990, 61, 326-330. [CrossRef]

17. Christiansen, C.L. The effects of hip and ankle stretching on gait function of older people. Arch. Phys. Med. Rehabil. 2008, 89, 1421-1428. [CrossRef]

18. Watt, J.; Jackson, K.; Franz, J.; Dicharry, J.; Evans, J.; Kerringan, D. Effect of a supervised hip flexor stretching program on gait in frail elderly patients. PMER 2011, 3, 330-335.

19. Regensteiner, J.; Gardner, A.; Hiatt, W. Exercise testing and exercise rehabilitation for patients with peripheral arterial disease: Status in 1997. Vasc. Med. 1997, 2, 147-155. [CrossRef]

20. Gibellini, R.; Ferrari, B.; Zambelli, M.; Fanello, M. La riabilitazione in Angiologia. Phlebologie-Paris 1997, $50,455$.

21. Mayorga-Vega, D.; Merino-Marban, R.; Viciana, J. Criterion-Related Validity of Sit-and-Reach Tests for Estimating Hamstring and Lumbar Extensibility: A Meta-Analysis. J. Sports Sci. Med. 2014, 13, 1-14. [PubMed]

22. Bohannon, R.; Tiberio, D.; Zito, M. Effect of five minute stretch on ankle dorsiflexion range of motion. J. Phys. Ther. Sci. 1994, $6,1-8$.

23. Hamburg, N.M.; Balady, G.J. Exercise rehabilitation in peripheral artery disease: Functional impact and mechanisms of benefits. Circulation 2011, 123, 87-97. [CrossRef] [PubMed]

24. Bisconti, A.V.; Cè, E.; Longo, S.; Venturelli, M.; Coratella, G.; Limonta, E.; Doria, C.; Rampichini, S.; Esposito, F. Evidence for improved systemic and local vascular function after long-term passive static stretching training of the musculoskeletal system. $J$. Physiol. 2020, 598, 3645-3666. [CrossRef]

25. Hortobágyi, T.; Lesinski, M.; Gäbler, M.; VanSwearingen, J.M.; Malatesta, D.; Granacher, U. Effects of Three Types of Exercise Interventions on Healthy Old Adults' Gait Speed: A Systematic Review and Meta-Analysis. Sport. Med. 2015, 45, 1627-1643. [CrossRef]

26. Zakari, M.; Alsahly, M.; Koch, L.G.; Britton, S.L.; Katwa, L.C.; Lust, R.M. Are There Limitations to Exercise Benefits in Peripheral Arterial Disease? Front. Cardiovasc. Med. 2018, 5, 173. [CrossRef] 